\title{
Approximation theorems of a solution of amperometric enzymatic reactions based on Green's fixed point normal-S iteration
}

\author{
Khanitin Muangchoo-in 1,2, Kanokwan Sitthithakerngkiet ${ }^{3}$, Parinya Sa-Ngiamsunthorn ${ }^{2,4}$ and \\ Poom Kumam ${ }^{1,45^{*}}$ (D)
}

\author{
"Correspondence: \\ poom.kum@kmutt.ac.th \\ ${ }^{1}$ Fixed Point Research Laboratory, \\ Fixed Point Theory and Applications \\ Research Group, Center of \\ Excellence in Theoretical and \\ Computational Science (TaCS-CoE) \\ Faculty of Science, King Mongkut's \\ University of Technology Thonburi \\ (KMUTT), 126 Pracha Uthit Rd., Bang \\ Mod, Thung Khru, Bangkok 10140, \\ Thailand \\ ${ }^{4}$ Center of Excellence in Theoretical \\ and Computational Science \\ (TaCS-CoE), Faculty of Science, King \\ Mongkut's University of Technology \\ Thonburi (KMUTT), 126 Pracha Uthit \\ Rd., Bang Mod, Thung Khru, \\ Bangkok 10140, Thailand \\ Full list of author information is \\ available at the end of the article
}

\begin{abstract}
In this paper, the authors present a strategy based on fixed point iterative methods to solve a nonlinear dynamical problem in a form of Green's function with boundary value problems. First, the authors construct the sequence named Green's normal-S iteration to show that the sequence converges strongly to a fixed point, this sequence was constructed based on the kinetics of the amperometric enzyme problem. Finally, the authors show numerical examples to analyze the solution of that problem.
\end{abstract}

Keywords: Fixed point iteration; Green's function; Enzymatic reaction; Boundary value problems

\section{Introduction}

The development of a mathematical model based on diffusion has received a great deal of attention in recent years, many scientist and mathematician have tried to apply basic knowledge about the differential equation and the boundary condition to explain and approximate the diffusion and reaction model [1-11].

In 2017, Abukhaled and Khuri [12] solved a solution of amperometric enzymatic reaction based on Green's function by using the fixed point iteration

$$
\frac{\partial^{2} s}{\partial x^{2}}-\frac{K s}{1+\alpha s}=0, \quad 0<s \leq 1,
$$

subject to

$$
s^{\prime}(0)=0, \quad s(0)=a, \quad \text { and } \quad s(1)=b .
$$

They defined an operator based on the Picard iteration and proved that the operator is a contraction mapping that shows the sequence convergence with regard to Banach's theorem.

(c) The Author(s) 2021. This article is licensed under a Creative Commons Attribution 4.0 International License, which permits use, sharing, adaptation, distribution and reproduction in any medium or format, as long as you give appropriate credit to the original author(s) and the source, provide a link to the Creative Commons licence, and indicate if changes were made. The images or other third party material in this article are included in the article's Creative Commons licence, unless indicated otherwise in a credit line to the material. If material is not included in the article's Creative Commons licence and your intended use is not permitted by statutory regulation or exceeds the permitted use, you will need to obtain permission directly from the copyright holder. To view a copy of this licence, visit http://creativecommons.org/licenses/by/4.0/. 
Theorem Banach ([13]) Let $(M, d)$ be a complete metric space and $P: M \rightarrow M$ be Banach's contraction map (that is, there exists $a \in[0,1)$ such that

$$
d(P x, P y) \leq a d(x, y)
$$

for all $x, y \in M$. Then $P$ has a unique fixed point $p \in M$. Furthermore, for each $x_{0} \in M$, the sequence $\left\{x_{n}\right\}$ defined by

$$
x_{n+1}=P x_{n}
$$

for each $n \geq 0$ converges to the fixed point $p$.

In 2018, Khuri and Louhichi [14] presented a new numerical approach for the numerical solution of boundary value problems. The algorithm is defined in terms of Green's function into the Ishikawa fixed point iteration [15]

$$
\left\{\begin{array}{l}
x_{0} \in M, \\
y_{n}=\left(1-\beta_{n}\right) x_{n}+\beta_{n} P x_{n}, \\
x_{n+1}=\left(1-\alpha_{n}\right) x_{n}+\alpha_{n} P y_{n}, \quad \forall n \in \mathbb{N},
\end{array}\right.
$$

where $\left\{\beta_{n}\right\}$ and $\left\{\alpha_{n}\right\}$ are sequences in $[0,1]$. Note that the step of $y_{n}$ is called Mann's iteration [16].

Further, the converge theorem was proved by using the theorem of Berinde [17].

Theorem Berinde Let $M$ be an arbitrary Banach space, $K$ is a closed convex subset of $M$ and $P: K \rightarrow K$, which the operator satisfies the Zamfirescu operator. Let $\left\{x_{n}\right\}_{n=0}^{\infty}$ be an Ishikawa iteration and $x_{0} \in K$, where $\{\alpha\}$ and $\{\beta\}$ are sequences of positive numbers in $[0,1]$ with $\left\{\beta_{n}\right\}$ satisfying $\sum_{n=0}^{\infty} \beta_{n}=\infty$. Then $\left\{x_{n}\right\}_{n=0}^{\infty}$ strongly converges to the fixed point of $P$.

The above operator is sometimes called Zamfirescu operator [18].

Theorem Zamfirescu Let $(M, d)$ be a complete metric space and $P: M \rightarrow M$ be a map for there exist the real numbers $a_{1}, a_{2}$, and $a_{3}$ satisfying $0 \leq a_{1}<1,0 \leq a_{2}, a_{c}<0.5$ such that, for each pair $x, y$ in $M$, at least one of the following is true:

$\left(z_{1}\right) d(P x, P y) \leq a_{1} d(x, y)$

$\left(z_{2}\right) d(P x, P y) \leq a_{2}[d(x, P x)+d(y, P y)]$

$\left(z_{3}\right) d(P x, P y) \leq a_{3}[d(x, P y)+d(y, P x)]$.

Then $P$ has a unique fixed point $p$ and the sequence $\left\{x_{n}\right\}_{n=0}^{\infty}$ defined by Picard iteration

$$
x_{n+1}=P x_{n}, \quad n=0,1,2, \ldots
$$

converges to $p$ for any $x_{0} \in M$.

In this paper, the authors use the motivation above to construct Green's normal-S iteration based on the sequence of normal-S iteration of Sahu [19]. Let $K$ be a convex subset of 
the normed space $M$ and a nonlinear mapping $P$, the sequence $\left\{x_{n}\right\}$ in $K$ is call normal-S if it is defined by

$$
\left\{\begin{array}{l}
x_{0} \in M, \\
y_{n}=\left(1-\beta_{n}\right) x_{n}+\beta_{n} P x_{n}, \\
x_{n+1}=P\left(y_{n}\right)
\end{array}\right.
$$

for each $n \geq 1$, where $\left\{\beta_{n}\right\}$ is the sequence in $[0,1]$.

The proof of the convergence theorem is based on Berinde's idea. Finally, the authors use the sequence to approximate problem (1) subject to (2) by showing a numerical example.

\section{Preliminaries}

\subsection{The mathematical model}

Diffusion equations were presented by a mathematical model related to MichaelisMenten kinetics (4) of the enzymatic reaction

$$
E+S \quad \Longleftrightarrow \quad E S \rightarrow E+P
$$

where $E$ is an enzyme, $S$ is a substrate, $E S$ is a complex between enzyme and substrate, and $P$ is a product of reaction.

In biochemistry, the enzyme kinetics in $n$-dimension $\Omega$ is modeled by the reactiondiffusion equation [20]

$$
\frac{\partial S}{\partial t}=D_{S} \nabla(\nabla S)-v(t, X), \quad X \in \Omega,
$$

where $D_{S}$ is the diffusion coefficient of a substrate and $v$ is the initial reaction velocity. By using the Michaelis-Menton hypothesis, the velocity $v$ for simple reaction processes without competitive inhibition is given by [20,21]

$$
v(t, X)=\frac{K S}{1+S / K_{M}},
$$

where $K=k_{2} E_{0} / K_{M}$ represents a pseudo first order, in which $k_{2}$ is the unimolecular rate constant, $E_{0}$ is the total amount of enzymes, and $K_{M}$ is the Michaelis constant. The onedimensional form of (5) is given by

$$
\frac{\partial S}{\partial t}=D_{S} \frac{\partial^{2} S}{\partial X^{2}}-\frac{K S}{1+S / K_{M}}, \quad X \in \Omega,
$$

with the initial condition given by

$$
S(0, X)=S_{0}(X)
$$

By introducing the parameters

$$
s=\frac{S}{K S^{\infty}}, \quad x=\frac{X}{L}, \quad \tau=\frac{D_{S}}{L^{2}}, \quad K=\frac{k L^{2}}{D_{S}}=\phi^{2}, \quad \alpha=\frac{k S^{\infty}}{K_{M}},
$$


we obtain the nonlinear reaction-diffusion equation at steady state

$$
\frac{\partial^{2} s}{\partial x^{2}}-\frac{K s}{1+\alpha s}=0, \quad 0<s \leq 1,
$$

where $S^{\infty}$ is the substrate concentration in bulk solution $\left(\mathrm{mol} \mathrm{dm}^{-3}\right), \phi^{2}$ is the Thiele modulus.

\subsection{Green's function}

Consider the second order differential equation decomposed into a linear term $\operatorname{Li}[y]$ and a nonlinear term $f\left(t, y, y^{\prime}\right)$ as follows:

$$
\operatorname{Li}[y] \equiv y^{\prime \prime}=f\left(t, y, y^{\prime}\right)
$$

subject to the boundary conditions

$$
\left\{\begin{array}{l}
\mathrm{BC}_{a}[y] \equiv a_{0} y(a)+a_{1} y^{\prime}(a)=\alpha, \\
\mathrm{BC}_{b}[y] \equiv b_{0} y(b)+b_{1} y^{\prime}(b)=\beta,
\end{array}\right.
$$

where $a \leq t \leq b$. Bernfeld and Lakshmikantham [22] presented the existence and uniqueness theorems for solutions of (11).

The Green's function $G(t, s)$ corresponding to the linear term $\operatorname{Li}[y]$ is defined as the solution of the following boundary value problem:

$$
\operatorname{Li}[G(t, s)]=\delta(t-s), \quad \mathrm{BC}_{a}[G(t, s)]=\mathrm{BC}_{b}[G(t, s)]=0,
$$

and has the piecewise form

$$
G(t, s)= \begin{cases}c_{1} y_{1}+c_{2} y_{2}, & a \leq t<s, \\ d_{1} y_{1}+d_{2} y_{2}, & s<t \leq b,\end{cases}
$$

where $y_{1}$ and $y_{2}$ form a fundamental set of solutions for $\operatorname{Li}[y]=0$. The unknowns could be found using the homogeneous conditions given in (12) and the fact that the Green's function is continuous and its first derivative has a unit jump discontinuity. More precisely, the constants are determined using the following properties:

A. $G$ satisfies the corresponding homogeneous boundary conditions

$$
\mathrm{BC}_{a}[G(t, s)]=\mathrm{BC}_{b}[G(t, s)]=0
$$

B. $G$ is continuous at $t=s$, i.e.,

$$
c_{1} y_{1}(s)+c_{2} y_{2}(s)=d_{1} y_{1}(s)+d_{2} y_{2}(s)
$$

C. $G^{\prime}$ has a unit jump discontinuity at $t=s$, i.e.,

$$
d_{1} y_{1}^{\prime}(s)+d_{2} y_{2}^{\prime}(s)-c_{1} y_{1}^{\prime}(s)-c_{2} y_{2}^{\prime}(s)=1 .
$$


A particular solution to $y^{\prime \prime}=f\left(t, y, y^{\prime}, y^{\prime \prime}\right)$ is expressed in terms of $\mathrm{G}$ and is given by the following structure:

$$
u_{p}=\int_{a}^{b} G(t, s) f\left(s, u_{p}, u_{p}^{\prime}\right) d s
$$

We construct the Green's function for the differential operator $\operatorname{Li}[y]=y^{\prime \prime}=0$, which has two linearly independent solutions $y_{1}(t)=1$ and $y_{2}(t)=t$. From (14), the Green's function will have the form

$$
G(t, s)= \begin{cases}c_{1}+c_{2} t, & 0 \leq t \leq s \\ d_{1}+d_{2} t, & s \leq t \leq 1\end{cases}
$$

where the unknowns are found by the properties $\mathrm{A}, \mathrm{B}$, and $\mathrm{C}$ listed above. To find the homogeneous boundary conditions, we have

$$
c_{1}=0, \quad d_{1}+d_{2}=0 .
$$

$G(t, s)$ is continuous and $G^{\prime}(t, s)$ discontinues at $t=s$ then

$$
\left\{\begin{array}{l}
c_{1}+c_{2} s=d_{1}+d_{2} s, \\
c_{2}-d_{2}=1 .
\end{array}\right.
$$

From (19)-(21), we obtain the following Green's function:

$$
G(t, s)= \begin{cases}s(1-t), & 0 \leq s \leq t \\ t(1-s), & t \leq s \leq 1\end{cases}
$$

\subsection{Green's normal-S iteration}

Applying the Green's function to the normal-S iterative method, we recall the following differential equation:

$$
\operatorname{Li}[y]+\operatorname{No}[y]=f(t, y)
$$

where $\operatorname{Li}[u]$ is a linear operator in $y, \operatorname{No}[y]$ is a nonlinear operator in $y$, and $f(t, y)$ is a linear or nonlinear function in $y$. Let $y_{p}$ be a particular solution of (23). We define the linear integral operator in terms of the Green's function and the particular solution $y_{p}$ as follows:

$$
K\left[y_{p}\right]=\int_{a}^{b} G(t, s) \operatorname{Li}\left[y_{p}\right] d s .
$$

Here, $G$ is the Green's function corresponding to the linear differential operator $\operatorname{Li}[y]$. For convenience, we set $y_{p}=v$. Adding and subtracting $\operatorname{No}[v]-f(s, v)$ from within the integral 
in (24) yield

$$
\begin{aligned}
K[v] & =\int_{a}^{b} G(t, s)(\operatorname{Li}[v]+\mathrm{No}[v]-f(s, v)) d s+\int_{a}^{b} G(t, s)(f(s, v)-\mathrm{No}[v]) d s \\
& =v+\int_{a}^{b} G(t, s)(\operatorname{Li}[v]+\mathrm{No}[v]-f(s, v)) d s .
\end{aligned}
$$

We then apply the normal-S fixed point iterative form

$$
\left\{\begin{array}{l}
v_{0} \in M \\
w_{n}=\left(1-\beta_{n}\right) v_{n}+\beta_{n} K\left[v_{n}\right] \\
v_{n+1}=K\left[w_{n}\right]
\end{array}\right.
$$

where $n \geq 0,\left(\beta_{n}\right)$ is a sequence of real numbers in $[0,1]$. That is,

$$
\left\{\begin{array}{l}
v_{0} \in M \\
w_{n}=\left(1-\beta_{n}\right) v_{n}+\beta_{n}\left[v_{n}+\int_{a}^{b} G(t, s)\left(\operatorname{Li}\left[v_{n}\right]+\operatorname{No}\left[v_{n}\right]-f\left(s, v_{n}\right)\right) d s\right] \\
v_{n+1}=w_{n}+\int_{a}^{b} G(t, s)\left(\operatorname{Li}\left[w_{n}\right]+\operatorname{No}\left[w_{n}\right]-f\left(s, w_{n}\right)\right) d s
\end{array}\right.
$$

which is reduced to

$$
\left\{\begin{array}{l}
v_{0} \in M, \\
w_{n}=v_{n}+\beta_{n} \int_{a}^{b} G(t, s)\left(\operatorname{Li}\left[v_{n}\right]+\operatorname{No}\left[v_{n}\right]-f\left(s, v_{n}\right)\right) d s, \\
v_{n+1}=w_{n}+\int_{a}^{b} G(t, s)\left(\operatorname{Li}\left[w_{n}\right]+\operatorname{No}\left[w_{n}\right]-f\left(s, w_{n}\right)\right) d s .
\end{array}\right.
$$

\section{Main results}

\subsection{Constructing the normal-S Green's iterative scheme}

Let $\operatorname{Li}[s]=\frac{\partial^{2} s}{\partial x^{2}}$ and $f(\alpha, K, s)=\frac{K s}{1+\alpha s}$, consider the enzyme substrate reaction equation, which takes the form of the following nonlinear equation:

$$
\operatorname{Li}[s]=s^{\prime \prime}(x)=f\left(x, s(x), s^{\prime}(x)\right)
$$

with boundary condition (2), then the required Green's function

$$
\operatorname{Li}[G(x, z)]=\delta(x-z)
$$

subject to the corresponding homogenous boundary conditions

$$
\left.\frac{d}{d x} G(x, z)\right|_{x=0}=0 \quad \text { and }\left.\quad G(x, z)\right|_{x=1}=0 .
$$

Using boundary condition (32) in Green's function (19) then $G(x, z)$, we obtain the equations

$$
c_{2}=0 \text { and } \quad d_{1}+d_{2}=0 .
$$


The continuity of $G$ implies that

$$
c_{2} z+c_{1}=d_{2} z+d_{1}
$$

and $\frac{d}{d x} G(x, z)$ jump discontinuity implies that

$$
d_{2}-c_{2}=1
$$

Hence,

$$
G(x, z)= \begin{cases}z-1, & 0 \leq x \leq z \\ x-1, & z \leq x \leq 1\end{cases}
$$

From (25), we introduce the following continuous functions on $[0,1]$ into itself:

$$
P_{G}\left(s_{n}\right)=s_{n}+\int_{0}^{1} G(x, z)\left(s_{n}^{\prime \prime}(z)-f\left(\alpha, K, s_{n}\right)\right) d z
$$

then equations (27)-(29) become

$$
\left\{\begin{array}{l}
s_{0} \in M \\
w_{n}=\left(1-\beta_{n}\right) s_{n}+\beta_{n} P_{G}\left(s_{n}\right) \\
s_{n+1}=P_{G}\left(w_{n}\right)
\end{array}\right.
$$

\subsection{Convergence theorems}

In Theorem 1 we show that the operator $P_{G}$ is a contraction mapping, and in Theorem 2 we show that if the operator $P$ satisfies condition $Z$, then the sequence $\left\{s_{n}\right\}_{n=0}^{\infty}$ defined by normal-S (29) converges strongly to the fixed point of $P$.

Theorem 1 Assume that the function $f$, which appears in the definition of the operator $P_{G}$, is such that

$$
C=C_{c}<1,
$$

where $C_{c}=\max _{x \in[0,1]}\left|f^{\prime}(s(x))\right|$. Then $P_{G}$ is a contraction, and hence the sequence $\left\{s_{n}\right\}$ is defined by normal-S iteration (29).

Proof Performing integration by parts in equations (29), (36)-(38), the product is

$$
P_{G}(s)=s-\int_{0}^{1} G(x, z) f\left(z, s, s^{\prime}\right) d z,
$$


where $s=w_{n}$ of (38). Thus

$$
\begin{aligned}
\left|P_{G}(s)-P_{G}(v)\right| & =\left|\int_{0}^{1} G(x, z)\left[f\left(z, v, v^{\prime}\right)-f\left(z, s, s^{\prime}\right)\right] d z\right| \\
& \leq\left(\int_{0}^{1}|G(x, z)| d z\right)\left(\int_{0}^{1}\left|f\left(z, v, v^{\prime}\right)-f\left(z, s, s^{\prime}\right)\right| d z\right) \\
& \leq\left(\int_{0}^{1}\left|f\left(z, v, v^{\prime}\right)-f\left(z, s, s^{\prime}\right)\right| d z\right) .
\end{aligned}
$$

By applying the mean value theorem for $f(s)$ and using the condition that $C_{c}=$ $\max _{x \in[0,1]}\left|f^{\prime}(s(x))\right|$, we consider the last inequality

$$
\begin{aligned}
\left|P_{G}(s)-P_{G}(v)\right| & \leq \max _{x \in[0,1]}|f(v(x))-f(s(x))| \\
& \leq C_{c}\|s-v\|,
\end{aligned}
$$

where $\|s-v\|=\max _{x \in[0,1]}|s(x)-v(x)|$ and $C=C_{c}<1$. So, we obtain the following:

$$
\left\|P_{G}(s)-P_{G}(v)\right\| \leq C\|s-v\|
$$

such that $0 \leq C<1$. Hence $P_{G}$ is a contraction mapping.

Theorem 2 Let $M$ be an arbitrary Banach space, $K$ be a closed convex subset of $M$, and $P: K \rightarrow K$ be an operator satisfying the condition of Zamfirescu. Let $\left\{s_{n}\right\}_{n=0}^{\infty}$ be defined by normal-S (3) and $s_{0} \in K$, where $\left\{\beta_{n}\right\}$ is a sequence in $[0,1]$. Then $\left\{s_{n}\right\}_{n=0}^{\infty}$ converges strongly to the fixed point of $P$.

Proof By Zamfirescu's theorem, we know that $P$ has a unique fixed point in $K$ that is $p$. Consider $s, m \in K$. Since $P$ is a Zamfirescu operator, at least one of conditions $\left(z_{1}\right),\left(z_{2}\right)$, and $\left(z_{3}\right)$ is satisfied. If $\left(z_{2}\right)$ holds, then

$$
\begin{aligned}
\|P s-P m\| & \leq a_{2}[\|s-P s\|+\|m-P m\|] \\
& \leq b[\|s-P s\|+\|m-s\|+\|s-P s\|+\|P s-P m\|],
\end{aligned}
$$

so

$$
\left(1-a_{2}\right)\|P s-P m\| \leq a_{2}\|s-m\|+2 a_{2}\|s-P s\|
$$

from $0 \leq a_{2}<1$

$$
\|P s-P m\| \leq \frac{a_{2}}{1-a_{2}}\|s-m\|+\frac{2 a_{2}}{1-a_{2}}\|s-P s\| .
$$

Similarly, if $\left(z_{3}\right)$ holds

$$
\|P s-P m\| \leq \frac{a_{3}}{1-a_{3}}\|s-m\|+\frac{2 a_{3}}{1-a_{3}}\|s-P s\| .
$$


Denote $\delta=\max \left\{a_{1}, \frac{a_{2}}{1-a_{2}}, \frac{a_{3}}{1-a_{3}}\right\}$. Then we have $0 \leq \delta<1$ and get

$$
\|P s-P m\| \leq \delta\|s-m\|+2 \delta\|s-P s\| .
$$

The sequence $\left\{s_{n}\right\}_{n=0}^{\infty}$ is defined by normal-S iteration (3) and $s_{0} \in K$, by (42) we get

$$
\left\|s_{n+1}-p\right\|=\left\|P p-P m_{n}\right\| \leq \delta\left\|m_{n}-p\right\| .
$$

Consider again

$$
\begin{aligned}
\left\|s_{n}-p\right\| & =\left\|\left(1-\beta_{n}\right) s_{n}+\beta_{n} P s_{n}-p\right\| \\
& =\left\|\left(1-\beta_{n}\right)\left(s_{n}-p\right)+\beta_{n}\left(P s_{n}-p\right)\right\| \\
& =\left(1-\beta_{n}\right)\left\|s_{n}-p\right\|+\beta_{n}\left\|P s_{n}-p\right\| .
\end{aligned}
$$

By (42) again,

$$
\left\|p-P s_{n}\right\| \leq \delta\left\|s_{n}-p\right\| .
$$

So, we have

$$
\begin{aligned}
\left\|s_{n+1}-p\right\| & \leq \delta\left\|m_{n}-p\right\| \\
& \leq \delta\left[\left(1-\beta_{n}\right)\left\|s_{n}-p\right\|+\beta_{n} P s_{n}-p \|\right] \\
& \leq \delta\left[\left(1-\beta_{n}\right)\left\|s_{n}-p\right\|+\beta_{n} \delta\left\|s_{n}-p\right\|\right] \\
& =\delta\left(1-\beta_{n}+\beta_{n} \delta\right)\left\|s_{n}-p\right\|,
\end{aligned}
$$

by induction

$$
\left\|s_{n+1}-p\right\| \leq \prod_{k=0}^{n} \delta\left(1-\beta_{k}+\beta_{k} \delta\right)\left\|s_{0}-p\right\| \quad \text { for } n=0,1,2, \ldots
$$

From $\delta\left(1-\beta_{k}+\beta_{k} \delta\right)<1$,

$$
\lim _{n \rightarrow \infty} \prod_{k=0}^{n} \delta\left(1-\beta_{k}+\beta_{k} \delta\right)=0
$$

which implies

$$
\lim _{n \rightarrow \infty}\left\|s_{n+1}-p\right\|=0 .
$$

Therefore $\left\{s_{n}\right\}_{n=0}^{\infty}$ converges strongly to the fixed point of $P$. This is completes the proof.

\section{Numerical examples}

In the first example, we show a simple example to compare the solution with three iterative methods to explain the convergence of the sequences. In the last example, we present the main example to analyze the main problem (10). 
Example 1 Consider the following differential equation $x(t)$ :

$$
x^{\prime \prime}(t)=\frac{3}{2} x(t)^{2},
$$

where $0 \leq t \leq 1$ and subject to

$$
x(0)=4, \quad x(1)=1 .
$$

The exact solution is $x(t)=\frac{4}{(1+t)^{2}}$. The initial iterate satisfies $x^{\prime \prime}=0$ and boundary conditions (47). This $x_{0}=4-3 t$. By normal-S Green's iteration (29),

$$
\begin{aligned}
& w_{n}=x_{n}+\beta_{n} \int_{0}^{t} s(1-t)\left(x_{n}^{\prime \prime}(s)-\frac{3}{2} x(s)^{2}\right) d s+\beta_{n} \int_{t}^{1} t(1-s)\left(x_{n}^{\prime \prime}(s)-\frac{3}{2} x(s)^{2}\right) d s, \\
& x_{n+1}=w_{n}+\int_{0}^{t} s(1-t)\left(w_{n}^{\prime \prime}(s)-\frac{3}{2} w(s)^{2}\right) d s+\int_{t}^{1} t(1-s)\left(w_{n}^{\prime \prime}(s)-\frac{3}{2} w(s)^{2}\right) d s .
\end{aligned}
$$

Table 1 shows the convergence step, Fig. 1 shows the convergence step and the error step of sequence $\left\{x_{n}\right\}$, which the error is calculated from $\left(\int_{a}^{b}\left|x_{n}-x_{\text {exact }}\right|^{2}\right)^{1 / 2}$.

Figure 1 shows a sequence of functions to compare three iterative methods. From the boundary condition, the value of problem starts at 4 and stops at 1 . The back line is the solution of function, while red, blue, and green lines are Mann, normal-S, and Ishikawa

Table 1 The convergence step of $\left\{x_{n}\right\}$ for example with $\alpha_{n}=0.1+\frac{0.000001}{n^{2}}$ and $\beta_{n}=0.005+\frac{0.0000001}{n^{2}}$

\begin{tabular}{lllll}
\hline$t$ & Exact solution & Mann & Ishikawa & Normal-S \\
\hline 0.0 & 3.92118419 & 3.92242687 & 1.30160882 & 3.92242687 \\
0.1 & 3.36671997 & 3.37519562 & 1.65351070 & 3.37519562 \\
0.2 & 2.82465927 & 2.83754278 & 2.35571598 & 2.83754278 \\
0.3 & 2.40370170 & 2.41804592 & 2.40900977 & 2.41804592 \\
0.4 & 2.07028621 & 2.08442445 & 2.14697656 & 2.08442445 \\
0.5 & 1.80172064 & 1.81466881 & 1.87174319 & 1.81466881 \\
0.6 & 1.58221589 & 1.59335578 & 1.63868670 & 1.59335578 \\
0.7 & 1.40051118 & 1.40941688 & 1.44510892 & 1.40941688 \\
0.8 & 1.24840048 & 1.25474044 & 1.28251199 & 1.25474044 \\
0.9 & 1.11978947 & 1.12326988 & 1.14342745 & 1.12326988 \\
1.0 & 1.01007550 & 1.01040682 & 1.02099028 & 1.01040682 \\
\hline
\end{tabular}
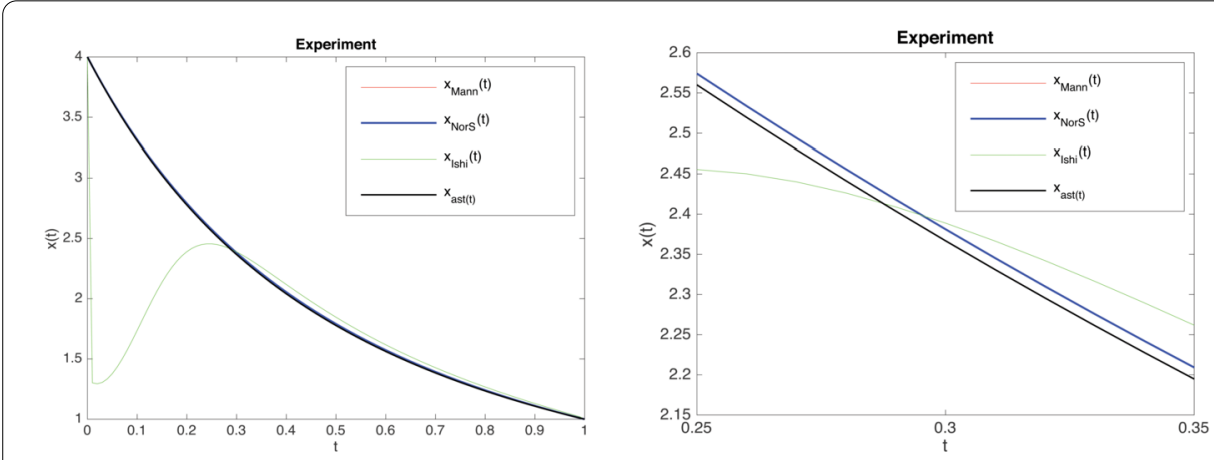

Figure 1 The convergence step of sequence $\left\{x_{n}\right\}$ with $\alpha_{n}=0.1+\frac{0.000001}{n^{2}}$ and $\beta_{n}=0.005+\frac{0.0000001}{n^{2}}$ 


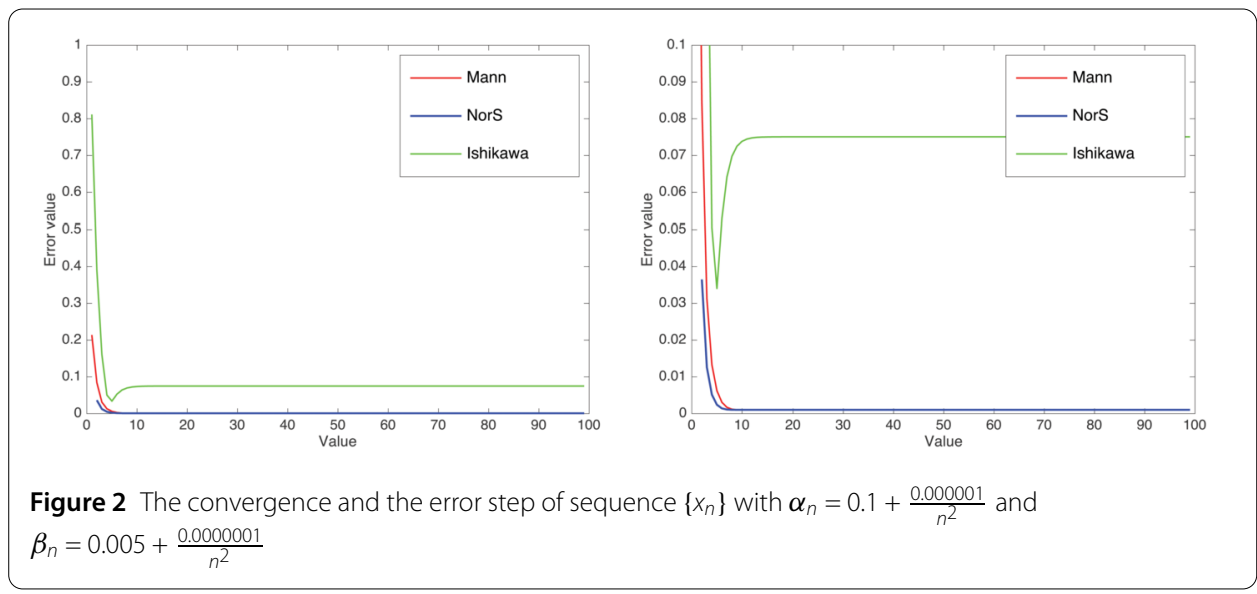

sequences, respectively. The figure concludes that Mann and normal-S are converging faster than Ishikawa and converging nearly to the solution of the function.

Figure 2 shows the error of three iterative sequences to compare the error value. Red, blue, and green lines mark Mann, Normal-S, and Ishikawa sequences, respectively. The figure concludes that normal-S sequence is decreasing to 0 faster than the error of Mann and Ishikawa sequences.

Example 2 Consider the differential equation (10)

$$
s^{\prime \prime}(x)=\frac{K s}{1+\alpha s},
$$

where $0 \leq x \leq 1$ and subject to

$$
s^{\prime}(0)=0, \quad s(0)=4 \quad \text { and } \quad s(1)=1 .
$$

The initial iterate satisfies $s^{\prime \prime}=0$ and the boundary conditions. Then $s_{0}=4-3 x$. By normal-S Green's iteration (29) and from (36), (37), and (38), the sequence is defined by

$$
\begin{aligned}
w_{n}= & s_{n}+\beta_{n} \int_{0}^{x}(z-1)\left(s_{n}^{\prime \prime}(z)-\frac{K s_{n}(z)}{1+\alpha s_{n}(z)}\right) d z \\
& +\beta_{n} \int_{x}^{1}(x-1)\left(s_{n}^{\prime \prime}(z)-\frac{K s_{n}(z)}{1+\alpha s_{n}(z)}\right) d z, \\
s_{n+1}= & w_{n}+\int_{0}^{x}(z-1)\left(w_{n}^{\prime \prime}(z)-\frac{K w_{n}(z)}{1+\alpha w_{n}(z)}\right) d z \\
& +\int_{x}^{1}(x-1)\left(w_{n}^{\prime \prime}(z)-\frac{K w_{n}(z)}{1+\alpha w_{n}(z)}\right) d z,
\end{aligned}
$$

where $K$ and $\alpha$ are constants of substrate concentration, and set $\beta_{n}=0.005+\frac{0.0000001}{n^{2}}$.

Table 2 and Fig. 3 show approximation of substrate concentration sequence $S(x)$ for different values of $\alpha$ and $K$.

Explanation of Fig. 3: Firstly, the error of normal-S sequence $S(x)$ compares with different values of $\alpha$ with $K=0.00001$, the error sequence of large $\alpha$ converges faster than that small $\alpha$. Secondly, the error of normal-S sequence $S(x)$ which compared by different values of $K$ with $\alpha=1000$, the error sequence of small $K$ converges faster than that of large $K$. 
Table 2 Approximation of substrate concentration sequence $S(x)$ for different values of $\alpha$ and $K$

\begin{tabular}{lrll}
\hline$K$ & \multicolumn{1}{l}{$\alpha$} & Value & Error \\
\hline 0.00001 & 1000 & 1.30229436 & 0.163849753 \\
& 10,000 & 1.31170037 & 0.0163837933 \\
& 100,000 & 1.30116511 & 0.00163148460 \\
0.00001 & 1000 & 1.30229436 & 0.163849753 \\
0.000005 & & 1.30114713 & 0.0819213023 \\
0.000000000001 & & 1.29999989 & $7.80258953 \mathrm{e}-06$ \\
\hline
\end{tabular}
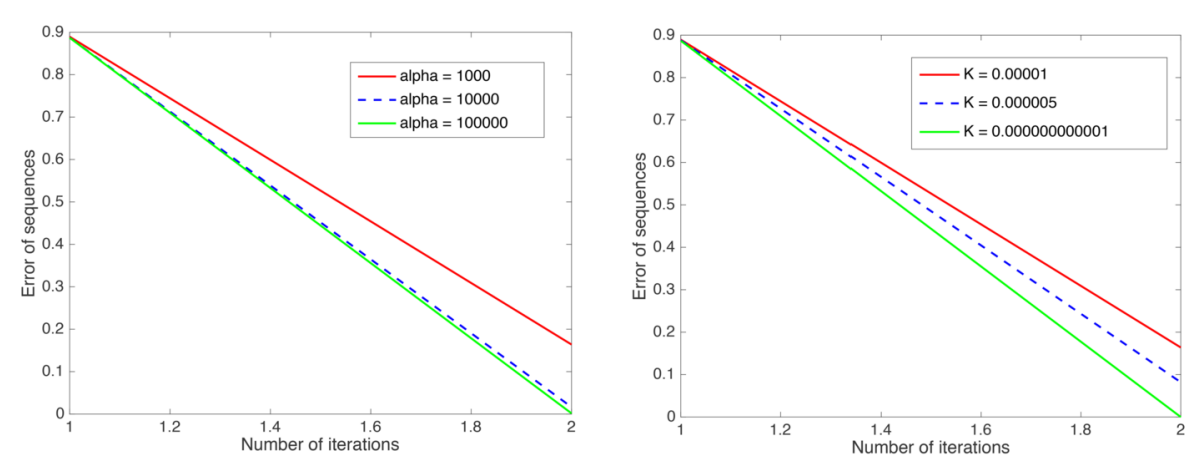

Figure 3 The error step of the sequence $\left\{s_{n}\right\}$ with different values of $\alpha$ with $K=0.00001$ and different values of $K$ with $\alpha=1000$

\section{Conclusion}

This paper presents a strategy based on fixed point iterative methods with normal-S iteration (38) to solve a nonlinear dynamical problem in a form of Green's function with boundary conditions used in Theorem 1 and Theorem 2 to guarantee the solution. Example 2 explains two constants $K$ and $\alpha$ in the nonlinear reaction-diffusion equation at steady state (1). Therefore, the values of $K$ must be small, while the values of $\alpha$ should be large, so the error value of sequence will converge to 0 faster than the other cases.

\section{Acknowledgements}

The authors would like to thank the Theoretical and Computational Science (TaCS) Center under Computational and Applied Science for Smart Innovation Cluster (CLASSIC), Faculty of Science, KMUTT and Intelligent and Nonlinear Dynamic Innovations Research Center, Department of Mathematics, Faculty of Applied Science, King Mongkut's University of Technology North Bangkok (KMUTNB), Bangkok, Thailand, contract no. KMUTNB-BasicR-64-22, for financial support.

\section{Funding}

This project was supported by the Faculty of Science, King Mongkut's University of Technology Thonburi (KMUTT) and the Faculty of Applied Science, King Mongkut's University of Technology North Bangkok (KMUTNB), Bangkok, Thailand. Contract no. KMUTNB-BasicR-64-22.

Availability of data and materials

Not applicable.

\section{Competing interests}

The authors declare that they have no competing interests.

Authors' contributions

All authors contributed equally in writing this article. All authors read and approved the final manuscript.

\section{Author details}

${ }^{1}$ Fixed Point Research Laboratory, Fixed Point Theory and Applications Research Group, Center of Excellence in

Theoretical and Computational Science (TaCS-CoE), Faculty of Science, King Mongkut's University of Technology Thonburi (KMUTT), 126 Pracha Uthit Rd., Bang Mod, Thung Khru, Bangkok 10140, Thailand. ${ }^{2}$ Department of Mathematics, Faculty of Science, King Mongkut's University of Technology Thonburi (KMUTT), 126 Pracha Uthit Rd., Bang Mod, Thung Khru, 
Bangkok 10140, Thailand. ${ }^{3}$ Intelligent and Nonlinear Dynamic Innovations Research Center, Department of Mathematics, Faculty of Applied Science, King Mongkut's University of Technology North Bangkok (KMUTNB), Bangkok 10800, Thailand. ${ }^{4}$ Center of Excellence in Theoretical and Computational Science (TaCS-CoE), Faculty of Science, King Mongkut's University of Technology Thonburi (KMUTT), 126 Pracha Uthit Rd., Bang Mod, Thung Khru, Bangkok 10140, Thailand. ${ }^{5}$ Department of Medical Research, China Medical University Hospital, China Medical University, Taichung 40402, Taiwan.

\section{Publisher's Note}

Springer Nature remains neutral with regard to jurisdictional claims in published maps and institutional affiliations.

Received: 29 July 2020 Accepted: 10 February 2021 Published online: 25 February 2021

\section{References}

1. Malvandi, A., Ganji, D.D.: A general mathematical expression of amperometric enzyme kinetics using He's variational iteration method with Pade approximation. J. Electroanal. Chem. 711, 32-37 (2013)

2. He, J.: Homotopy perturbation technique. Comput. Methods Appl. Mech. Eng. 17, 257-262 (1999)

3. Shanmugarajan, A., Alwarappan, S., Somasundaram, S., Lakshmanan, R.: Analytical solution of amperometric enzymatic reactions based on homotopy perturbation method. Electrochim. Acta 56, 3345-3352 (2001)

4. Agarwal, P., Baltaeva, U., Alikulov, Y.: Solvability of the boundary-value problem for a linear loaded integro-differential equation in an infinite three-dimensional domain. Chaos Solitons Fractals 140, 110108 (2020)

5. Zhou, H., Yang, L., Agarwal, P.: Solvability for fractional $p$-Laplacian differential equations with multipoint boundary conditions at resonance on infinite interval. J. Appl. Math. Comput. 53, 51-76 (2017)

6. Kaur, D., Rakshit, M., Agarwal, P., Chand, M.: Fractional calculus involving $(p, q)$-Mathieu type series. Appl. Math. Nonlinear Sci. 5(2), 15-34 (2020)

7. Agarwal, P., Attary, M., Maghasedi, M., Kumam, P.: Solving higher-order boundary and initial value problems via Chebyshev-spectral method: application in elastic foundation. Symmetry 12, 987 (2020)

8. Agarwal, P., Jleli, M., Samet, B.: The class of JS-contractions in Branciari metric spaces. Fixed Point Theory Metric Spaces 1, 79-87 (2018)

9. Agarwal, P., Jleli, M., Samet, B.: A coupled fixed point problem under a finite number of equality constraints. Fixed Point Theory Metric Spaces 1, 123-138 (2018)

10. Agarwal, P., Jleli, M., Samet, B.: JS-metric spaces and fixed point results. Fixed Point Theory Metric Spaces 1, 139-153 (2018)

11. Khatoon, S., Uddin, I., Baleanu, D.: Approximation of fixed point and its application to fractional differential equation J. Appl. Math. Comput. (2020). https://doi.org/10.1007/s12190-020-01445-1

12. Abukhaled, M., Khuri, S.A.: A semi-analytical solution of amperometric enzymatic reactions based on Green's function and fixed point iterative scheme. J. Electroanal. Chem. 792, 66-71 (2017)

13. Banach, S.: Sur les operations dans les ensembles abstraits et leur application aux equations integrals. Fundam. Math 3, 133-181 (1922)

14. Khuri, S.A., Louhichi, I.: A novel Ishikawa-Green's fixed point scheme for the solution of BVPs. Appl. Math. Lett. 82, 50-57 (2018)

15. Ishikawa, S.: Fixed points and iterations of non-expansive mappings in Banach spaces. Proc. Am. Math. Soc. 59, 65-71 (1976)

16. Mann, W.R.: Mean value methods in iteration. Proc. Am. Math. Soc. 4, 15-26 (1954)

17. Berinde, $\mathrm{V}:$ : On the convergence of the Ishikawa iteration in the class of quasi contractive operators. Acta Math. Univ. 73(1), 119-126 (2004)

18. Zamfirescu, T.: Fix point theorems in metric spaces. Arch. Math. (Basel) 23, 292-298 (1972)

19. Sahu, D.R.: Application of the S-iteration process to constrained minimization problem and split feasibility problem. Fixed Point Theory 12,187-204 (2013)

20. Pao, C.V.: Mathematical analysis of enzyme-substrate reaction diffusion in some biochemical systems. Nonlinear Anal., Theory Methods Appl. 4(2), 369-392 (1979)

21. Baronas, R., Ivanauskas, F., Kulys, J., Sapagovas, M.: Modeling of amperometric biosensors with rough surface of the enzyme membrane. J. Math. Chem. 34, 227-242 (2003)

22. Bernfeld, S.R., Lakshmikantham, V.: An Introduction to Nonlinear Boundary Value Problems. Academic Press, New York (1974)

\section{Submit your manuscript to a SpringerOpen ${ }^{\mathcal{O}}$ journal and benefit from:}

- Convenient online submission

- Rigorous peer review

- Open access: articles freely available online

- High visibility within the field

Retaining the copyright to your article

Submit your next manuscript at $>$ springeropen.com 